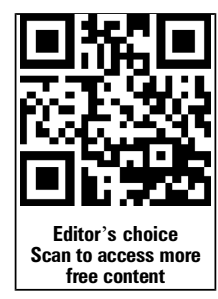

\title{
Asthma exacerbations during the first trimester of pregnancy and congenital malformations: revisiting the association in a large representative cohort
}

\author{
Lucie Blais, 1,2,3 Fatima-Zohra Kettani, ${ }^{1,2}$ Amélie Forget, ${ }^{1,2}$ \\ Marie-France Beauchesne, ${ }^{1,3,4,5}$ Catherine Lemière ${ }^{2,6}$
}

\begin{abstract}
${ }^{1}$ Faculty of Pharmacy, Université de Montréal, Montréal, Québec, Canada ${ }^{2}$ Research Center, Hôpital du Sacré-Cœur de Montréal, Montréal, Québec, Canada ${ }^{3}$ Endowment Pharmaceutical Chair AstraZeneca in Respiratory Health, Montréal, Québec, Canada

${ }^{4}$ Pharmacy Department, Centre Hospitalier Universitaire de Sherbrooke, Sherbrooke,

Québec, Canada

${ }^{5}$ Centre de Recherche Clinique (CR CHUS), Centre Hospitalier Universitaire de Sherbrooke, Sherbrooke, Québec, Canada ${ }^{6}$ Faculty of Medicine, Université de Montréal, Montréal, Québec, Canada
\end{abstract}

\section{Correspondence to} Dr Lucie Blais, Université de Montréal, Faculté de Pharmacie, C.P. 6128 , Succursale Centre-ville Montréal, Québec, Canada H3C 3J7;

lucie.blais@umontreal.ca

Received 1 December 2014 Accepted 24 March 2015

Published Online First 17 April 2015

\section{ABSTRACT}

Background We previously reported an increased prevalence of any congenital malformation among women experiencing moderate-to-severe asthma exacerbations during the first trimester of pregnancy, based on a study in which $90.1 \%$ of the cohort of women were social welfare recipients. This study re-examined the association between asthma exacerbations and congenital malformations in a new large representative cohort of asthmatic pregnant women.

Methods A cohort of 36587 pregnancies in asthmatic women was reconstructed from Québec Province administrative databases (1998-2009). Occurrences of asthma exacerbations during the first trimester of pregnancy were assessed and categorised into severe, moderate and no such exacerbations. For comparison, we also considered moderate and severe asthma exacerbations combined. Congenital malformations were identified using diagnoses recorded in the hospitalisation database. Generalised estimation equations were used to estimate adjusted ORs of congenital malformations.

Results The prevalence of any congenital malformation was $19.1 \%, 11.7 \%$ and $12.0 \%$ among women with severe, moderate and no such exacerbations during the first trimester, respectively. The adjusted OR for all malformations was $1.64(95 \% \mathrm{Cl} 1.02$ to 2.64$)$ when women with severe exacerbations were compared with those in the reference group, while no association was seen for moderate exacerbations. Also, no association was observed between cases of moderate and severe asthma exacerbations combined and any congenital malformation.

Conclusions Only severe asthma exacerbations were found to significantly increase the risk of congenital malformations in this representative study. Previous studies possibly overestimated the risk because they were based mainly on women at a lower socioeconomic status.

\section{INTRODUCTION}

Asthma is one of the most prevalent chronic diseases complicating pregnancy. ${ }^{1} 2$ Current asthma treatment guidelines highlight the importance and safety of the use of asthma medications during pregnancy as compared with the risk to the fetus of uncontrolled asthma. ${ }^{3}$ However, data regarding the association between asthma exacerbations during pregnancy and adverse fetal outcomes are

\section{Key messages}

What is the key question?

- What is the impact of asthma exacerbations during the first trimester of pregnancy on the prevalence of congenital malformations?

What is the bottom line?

- Only severe asthma exacerbations, that is, those requiring hospitalisation, were found to significantly increase the risk of congenital malformations, in this large representative study.

\section{Why read on?}

- This study provides additional evidence regarding the necessity of keeping asthma under control during pregnancy, using appropriate controller therapies to avoid exacerbations.

inconclusive ${ }^{4-23}$ and can be questioned on the basis of the small sample sizes and lack of power of numerous studies, the interstudy variations in the characteristics of study populations, the definition of exacerbations and their timing during pregnancy and the choice of the reference group (women without asthma or asthmatic women without exacerbations). ${ }^{4-23}$

For congenital malformations in particular, we identified 13 observational studies and one meta-analysis investigating the potential increase of congenital malformations among women with asthma exacerbations during pregnancy. ${ }^{7} 8 \quad 10 \quad 12-22$ Nine of the 13 studies, including five with small

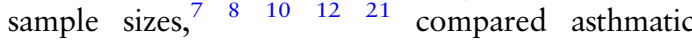
women with and without asthma exacerbations during pregnancy ${ }^{7} 81012-1621$ and reported ORs or risk ratios of congenital malformations (crude or adjusted) in the range of 1.0-1.7. Only one study, conducted by our group, reported a significant increased risk of any congenital malformation (adjusted OR=1.48, 95\% CI 1.04 to 2.09). ${ }^{15}$ Interestingly, this study, along with a second study from our group ${ }^{16}$ were the only studies that measured asthma exacerbations during the first trimester of pregnancy, that is, the most critical period for congenital malformations. Regarding the study
To cite: Blais $L$, Kettanif
Forget $A$, et al. Thorax

2015;70:647-652 
populations, the type of health insurance plan was not specified in six of the nine studies, ${ }^{7810121421}$ one study was conducted among women with private healthcare insurance, ${ }^{13}$ and our two studies were conducted among women with public drug insurance only. ${ }^{15} 16$ The meta-analysis, which included four of the nine studies, reported that asthma exacerbations during pregnancy were not associated with an increased prevalence of congenital malformation. ${ }^{23}$

Although we found a significant association between asthma exacerbations and any congenital malformation in our previous study, ${ }^{15}$ our findings were based on a cohort of women who were covered by the provincial public drug insurance plan, $90 \%$ of whom were recipients of social welfare. In addition, the definition of asthma exacerbations that was used in the study combined severe and moderate asthma exacerbations (see definitions below). Knowing that asthma might be more risky for congenital malformations among women with low socioeconomic status, ${ }^{24}$ one may wonder whether our previous significant result could be generalised to all asthmatic women, regardless of socioeconomic status. This study aimed therefore to re-examine the association between asthma exacerbations during the first trimester of pregnancy and the prevalence of congenital malformations in a large and representative cohort of women with asthma.

\section{MATERIALS AND METHODS}

\section{Data source and ethics considerations}

Data for the present study were retrieved from the Quebec Asthma and Pregnancy Database, which has been previously used to examine the association between asthma, asthma medications and congenital malformations. $^{24} 25$ This database, which was formed from the linkage of two administrative health databases from the province of Québec (Canada), the Maintenance et Exploitation des Données pour l'Étude de la Clientèle Hospitalière (MED-ECHO) and the Régie de l'assurance-maladie du Québec (RAMQ) databases, has been largely described in previous studies. Briefly, the MED-ECHO database contains information on all acute care hospitalisations, and the RAMQ database provides information on inpatient and ambulatory medical services claims, for all residents of Québec. The MED-ECHO and RAMQ databases contain a unique identifier, the individual's health insurance number, that serves as a link between them.

The Quebec Asthma and Pregnancy Database comprises all women who delivered between January 1990 and March 2010, and who had at least one asthma diagnosis recorded in the RAMQ or MED-ECHO databases in the 2 years prior to one or more of their deliveries, plus a fourfold larger random sample of other women who delivered during the same period. All pregnancies of the selected women with delivery between January 1990 and March 2010 were included in the database. Using the date of birth of the offspring recorded in the MED-ECHO database and the gestational age at birth, we retrospectively identified the date of the first day of the last menstrual period and the date of delivery for each pregnancy. The algorithms used to determine those dates have been formally evaluated and found to be highly valid. ${ }^{25}$ For each pregnancy included in the cohort, we identified the infants using the mother-child link provided in the RAMQ database; we had access to data related to hospitalisations and to medical services dispensed between January 1988 and March 2010, for all of the pregnant women and their infants.

An authorisation was obtained from the Commission d'accès à l'information $d u$ Québec before requesting and linking the information from the MED-ECHO and RAMQ databases.

\section{Study design}

A retrospective cohort design was used in this study. This cohort included pregnancies from the Québec Asthma and Pregnancy Database that met the following inclusion criteria: (1) delivery between January 1998 and March 2009; (2) gestation of 2045 weeks; (3) maternal age of 15-45 years at the onset of pregnancy and (4) pregnancy in a woman with active asthma. Asthma was defined as at least one asthma diagnosis (International Classification of Diseases (ICD)-9 code 493, except 493.2, or ICD-10 code J45) coded during a hospitalisation, or at least two medical claims with an asthma diagnosis within two consecutive years between 1988 and the delivery. ${ }^{26}$ This definition of an asthma case was validated in a cohort of men and women in the province of Ontario, Canada, and was found to have a sensitivity of $83.8 \%$ and a specificity of $76.5 \% .^{26}$ Among asthmatic women, asthma was considered to be active during pregnancy if there was at least one medical service for asthma recorded in the RAMQ or MED-ECHO databases within 2 years preceding the delivery. The exclusion criteria were as follows: (1) pregnancies with no data available for the offspring and (2) pregnancies with four or more births (including both live and stillbirths).

\section{Maternal asthma exacerbations}

The primary exposure of interest was the occurrence of asthma exacerbations during the first trimester of pregnancy (ie, during the first 14 weeks); exposure was categorised into three levels: (1) severe exacerbation: one or more hospitalisation with a primary or admission diagnosis of asthma; (2) moderate exacerbation: one or more emergency department (ED) visit for asthma, but no hospitalisation for asthma and (3) no hospitalisation and no ED visit for asthma (reference group). For purposes of comparison with prior studies, we also considered a two-level secondary exposure variable, in which moderate and severe asthma exacerbations were combined.

\section{Congenital malformations}

Based on the infant medical records for live births or the mothers' records for stillbirths, we identified all cases of congenital malformations at birth or during the first year of life based on the diagnostic codes of the ICD (prior to 2006: ICD-9 740-759; since 2006: ICD-10 Q01-Q99) recorded in the MED-ECHO database. Our list of ICD-9 diagnostic codes for congenital malformations was compared with the list provided by the Collaborative Perinatal Group, ${ }^{27}$ and its accuracy and completeness were verified by a geneticist from Montreal's Hopital Ste-Justine. The conversion of ICD-9 to ICD-10 codes was validated by a medical archivist from the Centre Hospitalier Universitaire de Sherbrooke.

As a first step, the geneticist classified the malformations as minor, major (life-threatening or causing major cosmetic defects) or undetermined (minor or major). In a second step, congenital malformations initially classified as undetermined were reclassified as major if there was at least one hospitalisation with an admission or a primary diagnosis of the malformation recorded in the MEDECHO database within the first year of life. Congenital malformation diagnoses recorded in Québec's administrative databases were found to have a positive predictive value of $82.2 \%$ for any malformation and $78.1 \%$ for major malformations among women with asthma. ${ }^{28}$ The primary outcome was any congenital malformation, and the secondary outcome was a major congenital malformation.

\section{Potential confounders}

Potential confounders included age at the start of pregnancy (classified as $<18,18-34$ and $>34$ years), drug insurance status 
at the start of pregnancy (publicly insured with social welfare, publicly insured without social welfare and privately insured), area of residence at the start of pregnancy (rural/urban/missing), multiple pregnancies (twins or triplets/singleton), chronic hypertension (yes/no), diabetes mellitus (yes/no) and epilepsy (yes/no). Chronic hypertension, diabetes mellitus and epilepsy were identified from diagnoses recorded in the RAMQ or MED-ECHO databases within 1 year of the onset of pregnancy or during the first trimester of pregnancy.

\section{Statistical analyses}

The characteristics of pregnancies and the prevalence of congenital malformations were compared between the three levels of exacerbation using descriptive statistics.

The association between maternal asthma exacerbations occurring during the first trimester of pregnancy and congenital malformations was evaluated using generalised estimation equation (GEE) models ${ }^{29}$ with a logit link. Two models (one for any malformations and the other for major congenital malformations) were used, both for the 3-level exacerbation variable and the 2-level exacerbation variable. The GEE models were used to estimate crude ORs and ORs adjusted for all confounding variables (and 95\% CIs). ${ }^{30}$ The models also accounted for the fact that some women had two or more pregnancies during the study period by considering correlations between the consecutive pregnancies in these women. All analyses were performed using SAS V.9.3 software (SAS Institute, Cary, North Carolina, USA).

\section{RESULTS}

The cohort consisted of 36587 pregnancies in women with active asthma. Among them, $110(0.3 \%)$ had a severe exacerbation and $1413(3.9 \%)$ had a moderate exacerbation in the first trimester of pregnancy. Table 1 shows the characteristics of the pregnancies according to the 3-level exacerbation variable.

The proportions of women aged $\leq 18$ years and having public drug insurance or living in a rural area were greater in the group of women who had a severe exacerbation than in the other two groups. Moreover, the proportions of women with multiple pregnancies, diabetes mellitus, epilepsy or chronic hypertension were greater in women with a severe exacerbation.

The prevalence of any congenital malformation was $19.1 \%$ in the severe exacerbation group, $11.7 \%$ in the moderate exacerbation group and $12.0 \%$ in the reference group; these values were $11.8 \%, 7.6 \%$ and $6.8 \%$, respectively, for major malformations (table 2).

Having a severe exacerbation in the first trimester of pregnancy was associated with a significant OR of 1.64 for any congenital malformation $(95 \%$ CI 1.02 to 2.64$)$ and a non-significant $\mathrm{OR}$ of 1.70 for a major congenital malformation (95\% CI 0.95 to 3.02), as compared with the reference group (table 3). No association was observed between moderate exacerbations and congenital malformations. Being the recipient of social welfare, living in a rural area, having multiple pregnancies or suffering from epilepsy also increased the prevalence of any malformation, and having diabetes mellitus increased the prevalence of major congenital malformations.

The adjusted regression models using the 2-level exacerbation variable revealed no association between moderate and severe asthma exacerbations combined and any congenital malformation $(\mathrm{OR}=1.02,95 \% \mathrm{CI} 0.87$ to 1.19$)$ or major congenital malformation $(\mathrm{OR}=1.15,95 \% \mathrm{CI} 0.95$ to 1.39$)$.

\section{DISCUSSION}

In this representative cohort, we found that severe maternal asthma exacerbations (ie, requiring hospitalisation for asthma) during the first trimester of pregnancy were significantly associated with an increased prevalence of congenital malformations, while moderate asthma exacerbations (ie, requiring an ED visit and no hospitalisation) were not. Also, we found no association between moderate and severe asthma exacerbations combined and the prevalence of congenital malformations.

We reviewed 13 observational studies and one meta-analysis that evaluated the impact of maternal asthma exacerbations on

Table 1 Characteristics of pregnancies according to the level of asthma exacerbation in the first trimester of pregnancy: severe (hospitalisation), moderate (ED visit and no hospitalisation) and reference group (neither ED visit or hospitalisation)

\begin{tabular}{|c|c|c|c|}
\hline Characteristics & $\begin{array}{l}\text { Hospitalisation } \\
\text { for asthma }(n=110)\end{array}$ & $\begin{array}{l}\text { ED visit and no } \\
\text { hospitalisation for asthma }(n=1413)\end{array}$ & $\begin{array}{l}\text { No hospitalisation and no } \\
\text { ED visit for asthma }(n=35064)\end{array}$ \\
\hline \multicolumn{4}{|l|}{ Age* (years) $^{*}$} \\
\hline$<18$ & $5(4.5)$ & $55(3.9)$ & $893(2.5)$ \\
\hline $18-34$ & $97(88.2)$ & $1264(89.5)$ & $30596(87.3)$ \\
\hline$>34$ & $8(7.3)$ & $94(6.6)$ & $3575(10.2)$ \\
\hline \multicolumn{4}{|l|}{ Drug insurance status* } \\
\hline Publicly insured women with social welfare & $32(29.1)$ & $324(22.9)$ & $5584(15.9)$ \\
\hline Publicly insured women without social welfare & $34(30.9)$ & $427(30.2)$ & $8184(23.4)$ \\
\hline Privately insured women & $44(40.0)$ & $662(46.9)$ & $21296(60.7)$ \\
\hline \multicolumn{4}{|l|}{ Area of residence* } \\
\hline Urban & $83(75.5)$ & $1146(81.1)$ & $29166(83.2)$ \\
\hline Rural & $26(23.6)$ & $257(18.2)$ & $5604(16.0)$ \\
\hline Missing & $1(0.9)$ & $10(0.7)$ & $294(0.8)$ \\
\hline Multiple pregnancy & $3(2.7)$ & $25(1.8)$ & $580(1.7)$ \\
\hline Diabetes mellitus $†$ & $10(9.1)$ & $25(1.8)$ & $794(2.3)$ \\
\hline Epilepsy† & $2(1.8)$ & $9(0.6)$ & $183(0.5)$ \\
\hline Chronic hypertensiont & 4 (3.6) & $38(2.7)$ & $903(2.6)$ \\
\hline
\end{tabular}


Table 2 Prevalence of congenital malformations according to the level of asthma exacerbation in the first trimester of pregnancy: severe (hospitalisation), moderate (ED visit and no hospitalisation) and reference group (neither ED visit nor hospitalisation)

\begin{tabular}{llll}
\hline & $\begin{array}{l}\text { Hospitalisation } \\
\text { for asthma } \\
(\mathrm{n}=110)\end{array}$ & $\begin{array}{l}\text { ED visit and no } \\
\text { hospitalisation } \\
\text { for asthma } \\
(\mathrm{n}=1413)\end{array}$ & $\begin{array}{l}\text { No } \\
\text { hospitalisation } \\
\text { and no ED visit } \\
\text { for asthma } \\
(\mathrm{n}=35064)\end{array}$ \\
\hline $\begin{array}{l}\text { Any congenital } \\
\text { malformation, } \mathrm{n}(\%)\end{array}$ & $21(19.1)$ & $166(11.7)$ & $4196(12.0)$ \\
$\begin{array}{l}\text { Major congenital } \\
\text { malformation, } \mathrm{n}(\%)\end{array}$ & $13(11.8)$ & $107(7.6)$ & $2384(6.8)$ \\
\hline
\end{tabular}

$E D$, emergency department.

congenital malformations, but found the results of these studies inconclusive, with ORs or risk ratios of 1.0-2.20 being significant in only three studies. ${ }^{8} 10^{12-23}$ One possible reason for the inconclusive results is related to the composition of control groups. For example, in four of the studies, two of which reported significant results, the reference group consisted of women without asthma, which therefore limits the capacities of these studies to isolate the impact of the exacerbations from the influence of the disease. ${ }^{17-20}$ Among the nine studies that used a reference group formed of asthmatic women without exacerbations, only one (conducted previously by our team) found a significant association between asthma exacerbations during the first trimester of pregnancy (defined as either a filled prescription of oral corticosteroids ( $\leq 14$ days) or an ED visit or hospitalisation for asthma) and any congenital malformation (adjusted $\mathrm{OR}=1.48$, 95\% CI 1.04 to 2.09$).{ }^{15}$ However, this study was based on a cohort formed only of women covered by public drug insurance, $90.1 \%$ of whom were receiving social welfare; therefore, the external validity of the results is questionable. ${ }^{15}$ Moreover, in that study, we used an outcome definition that combined all markers of asthma exacerbation, regardless of the level of severity of the exacerbation. ${ }^{15}$

In the present study, based on a representative cohort of pregnancies from women with asthma that included only $16 \%$ of women receiving social welfare, we found that the prevalence of any congenital malformation in the infants of women who had a severe asthma exacerbation in the first trimester of pregnancy was $64 \%$ greater than in the reference group, and no increased prevalence among women showing moderate exacerbations during the first trimester of pregnancy. In contrast to the results of our previous study, in which $90 \%$ of the cohort of women were receiving social assistance, this study was based on a more representative cohort, in which our definition of asthma exacerbations was refined by considering moderate and severe exacerbations separately, and thereby demonstrating that the prevalence of congenital malformations was increased only in pregnant women with severe exacerbations. So as to compare our current results with our previous findings, we also estimated the impact of moderate and severe asthma exacerbations combined on congenital malformations, and this time found no significant association. In light of these results, we suspect that part of the increased prevalence of congenital malformation observed in our previous study was due to the composition of the cohort, which consisted predominantly of women receiving social welfare. In fact, in another recent study, we found that the impact of maternal asthma on major congenital malformations was significantly greater in women receiving social welfare than in other women. ${ }^{24}$

It is noteworthy that in the present study, we had no access to drug data for approximately $70 \%$ of the pregnancies included in

Table 3 ORs describing the association between any and major congenital malformations and moderate and severe asthma exacerbations during the first trimester of pregnancy

\begin{tabular}{|c|c|c|c|c|}
\hline & \multicolumn{2}{|c|}{ Any congenital malformation } & \multicolumn{2}{|c|}{ Major congenital malformation } \\
\hline & Crude OR $(95 \% \mathrm{Cl})$ & Adjusted OR $(95 \% \mathrm{Cl})$ & Crude OR $(95 \% \mathrm{Cl})$ & Adjusted OR $(95 \% \mathrm{Cl})$ \\
\hline \multicolumn{5}{|l|}{ Level of asthma exacerbation } \\
\hline Severe exacerbations (hospitalisation for asthma) & 1.73 (1.07 to 2.78$)$ & 1.64 (1.02 to 2.64$)$ & 1.84 (1.03 to 3.28$)$ & $1.70(0.95$ to 3.02$)$ \\
\hline Moderate exacerbations (ED visit and no hospitalisation for asthma) & $0.98(0.83$ to 1.16$)$ & 0.97 (0.82 to 1.14$)$ & $1.12(0.92$ to 1.37$)$ & $1.10(0.90$ to 1.35$)$ \\
\hline Reference group (no hospitalisation and no ED visit for asthma) & Reference & Reference & Reference & Reference \\
\hline \multicolumn{5}{|l|}{ Age $^{*}$ (years) } \\
\hline$<18$ & 1.01 (0.83 to 1.24$)$ & $1.00(0.82$ to 1.22$)$ & $1.02(0.79$ to 1.31$)$ & $0.97(0.75$ to 1.25$)$ \\
\hline $18-34$ & Reference & Reference & Reference & Reference \\
\hline$>34$ & 0.97 (0.87 to 1.08$)$ & 0.96 (0.86 to 1.07$)$ & 1.07 (0.93 to 1.22$)$ & $1.05(0.92$ to 1.20$)$ \\
\hline \multicolumn{5}{|l|}{ Drug insurance status* } \\
\hline Publicly insured women without social welfare & 0.97 (0.89 to 1.05$)$ & 0.96 (0.88 to 1.04$)$ & $0.96(0.87$ to 1.06$)$ & 0.95 (0.86 to 1.06$)$ \\
\hline Publicly insured women with social welfare & 1.12 (1.03 to 1.22$)$ & 1.11 (1.02 to 1.21$)$ & 1.30 (1.17 to 1.45$)$ & 1.28 (1.15 to 1.43$)$ \\
\hline Privately insured women & Reference & Reference & Reference & Reference \\
\hline \multicolumn{5}{|l|}{ Area of residence* } \\
\hline Urban & Reference & Reference & Reference & Reference \\
\hline Rural & 1.16 (1.06 to 1.26$)$ & 1.16 (1.06 to 1.26$)$ & 1.15 (1.04 to 1.28$)$ & 1.16 (1.04 to 1.29$)$ \\
\hline Missing & 0.79 (0.54 to 1.16$)$ & 0.79 (0.54 to 1.16$)$ & 0.77 (0.46 to 1.28$)$ & 0.77 (0.47 to 1.28$)$ \\
\hline Multiple pregnancy & 2.39 (1.98 to 2.89$)$ & 2.40 (1.99 to 2.90$)$ & 3.14 (2.54 to 3.87$)$ & 3.13 (2.54 to 3.87 ) \\
\hline Diabetes mellitus $†$ & $1.19(0.97$ to 1.45$)$ & $1.14(0.94$ to 1.40$)$ & $1.36(1.06$ to 1.73$)$ & 1.27 (1.01 to 1.60$)$ \\
\hline Epilepsy† & 1.51 (1.04 to 2.20$)$ & 1.48 (1.01 to 2.15$)$ & 1.66 (1.05 to 2.63$)$ & 1.56 (0.99 to 2.47$)$ \\
\hline Chronic hypertensiont & $1.18(0.98$ to 1.43$)$ & 1.17 (0.97 to 1.41$)$ & 1.30 (1.04 to 1.64$)$ & 1.27 (0.99 to 1.62$)$ \\
\hline
\end{tabular}


the cohort, which precluded our use of oral corticosteroid use in our definition of asthma exacerbation. Indeed, the RAMQ database provides data on prescribed medications only for patients covered by the RAMQ's public drug insurance plan, which includes approximately $30 \%$ of women of childbearing age.

It has been reported that asthma exacerbations requiring a hospitalisation occur in about $6 \%$ of pregnant women. ${ }^{31}$ Exacerbations during pregnancy occur primarily in the later part of the second trimester, which might explain the lower prevalence $(0.3 \%)$ of hospitalisations for asthma observed in the present study as only the first trimester was examined here. Maternal asthma exacerbations, especially if severe, can plausibly be dangerous to the fetus because they can lead to impaired blood oxygenation and hypoxia in the fetus; an increasing body of evidence indicates that oxygen supply to the fetus in the first trimester is strongly regulated, and that hypoxia during this time results in abnormal fetal development. ${ }^{32}$ In addition, animal studies have shown that induced fetal hypoxia in mammalian and other animal embryos is associated with defects such as transverse limb reduction, cleft lip and heart defects. ${ }^{32}$

This study has some important strengths, mainly the representativeness of the population, the use of recognised and objective markers of asthma exacerbations ${ }^{33}$ and the large sample size, which allows for the assessment of exacerbations during the first trimester of pregnancy and the separation of moderate and severe exacerbations. In addition, using administrative databases, our data were collected prospectively and independently of the outcome, thus excluding the possibility of recall bias. Moreover, the asthma case definition used in the study and the congenital malformation diagnosis codes recorded in Québec's administrative databases were found to be highly valid.

There are also some limitations of the study to be taken into account when interpreting the results. As mentioned previously, because we did not have access to drug data for $70 \%$ of the pregnancies, we were not able to consider the use of oral corticosteroids in our definition of asthma exacerbation or to describe other components of asthma control, such as short-acting $\beta 2$-agonist use. The group of women without ED visits or hospitalisation for asthma might have shown mild asthma exacerbations that did not necessitate acute care, and because of this we may have underestimated the impact of moderate-to-severe asthma exacerbations on the prevalence of congenital malformations. In addition, we were not able to adjust the ORs for other known teratogenic drugs taken during the first trimester or for medications taken during hospitalisation. Women hospitalised for asthma are likely to have severe asthma, and it was therefore not possible in this study to differentiate between the impact of the severity of the disease and the impact of the severity of the exacerbation on the prevalence of congenital malformation. Finally, because of the nature of our observational design, it is possible that the study results were confounded by imbalances between the compared groups in variables that are not recorded in the databases but that are known to be associated with the prevalence of congenital malformations, such as obesity, alcohol use and cigarette smoking.

In conclusion, this large and representative cohort study showed that the prevalence of any congenital malformation was increased only for women with severe exacerbations requiring hospitalisation. Our previous findings on the association of moderate-to-severe asthma exacerbations and congenital malformations may have overestimated the risk, as the study was based on a cohort consisting mainly of women receiving social welfare. This study provides additional evidence on the necessity of keeping asthma under control during pregnancy, using adequate controller therapies, which are highly effective in reducing the risk of asthma-related exacerbations. ${ }^{34}$

Acknowledgements We thank the Régie de l'Assurance Maladie du Québec for assistance with the data. We are grateful to the Commission d'Accès à I'Information du Québec for authorising the study.

Contributors $L B, M-F B$ and $C L$ participated in the design of the study. AF helped with the analysis of data and statistics. F-ZK helped with the review of literature and the preparation of the first draft of the manuscript. All the authors revised and approved the final version of the manuscript.

Funding This study was funded by the Canadian Institutes of Health Research (MOP-97731).

Competing interests LB holds the AstraZeneca Chair in Respiratory Health; MFB co-holds the AstraZeneca Pharmaceutical Chair in Respiratory health.

Ethics approval The Ethics Committee of the Hôpital du Sacré-Coeur de Montréal.

Provenance and peer review Not commissioned; externally peer reviewed.

\section{REFERENCES}

1 Kwon HL, Triche EW, Belanger K, et al. The epidemiology of asthma during pregnancy: prevalence, diagnosis, and symptoms. Immunol Allergy Clin North Am 2006;26:29-62.

2 Charlton RA, Hutchison A, Davis KJ, et al. Asthma management in pregnancy. PLoS ONE 2013:8:e60247.

3 Busse W. NAEPP expert panel report. Managing asthma during pregnancy: recommendations for pharmacologic treatment-2004 update. J Allergy Clin Immunol 2005;115:34-46.

4 Firoozi $\mathrm{F}$, Lemiere $\mathrm{C}$, Beauchesne $\mathrm{MF}$, et al. Impact of maternal asthma on perinatal outcomes: a two-stage sampling cohort study. Eur J Epidemiol 2012;27:205-14.

5 Bakhireva LN, Schatz M, Jones KL, et al. Asthma control during pregnancy and the risk of preterm delivery or impaired fetal growth. Ann Allergy Asthma Immunol 2008; 101:137-43.

6 Bracken MB, Triche EW, Belanger K, et al. Asthma symptoms, severity, and drug therapy: a prospective study of effects on 2205 pregnancies. Obstet Gynecol 2003; 102:739-52.

7 SteniusAarniala BSM, Hedman J, Teramo KA. Acute asthma during pregnancy. Thorax 1996:51:411-14

8 Jana N, Vasishta K, Saha SC, et al. Effect of bronchial asthma on the course of pregnancy, labour and perinatal outcome. J Obstet Gynaecol 1995;21:227-32.

9 Schatz M, Zeiger RS, Hoffman CP, et al. Perinatal outcomes in the pregnancies of asthmatic women: a prospective controlled analysis. Am J Respir Crit Care Med 1995;151:1170-4.

10 Greenberger PA, Patterson R. The outcome of pregnancy complicated by severe asthma. Allergy Proc 1988;9:539-43.

11 Gordon M, Niswander KR, Berendes $\mathrm{H}$, et al. Fetal morbidity following potentially anoxigenic obstetric conditions. VII. Bronchial asthma. Am J Obstet Gynecol 1970;106:421-9

12 Fitzsimons R, Greenberger PA, Patterson R. Outcome of pregnancy in women requiring corticosteroids for severe asthma. J Allergy Clin Immunol 1986;78:349-53.

13 Schatz M, Zeiger RS, Harden $\mathrm{K}$, et al. The safety of asthma and allergy medications during pregnancy. J Allergy Clin Immunol 1997;100:301-6.

14 Schatz M, Dombrowski MP, Wise R, et al. The relationship of asthma medication use to perinatal outcomes. J Allergy Clin Immunol 2004;113:1040-5.

15 Blais $\mathrm{L}$, Forget $\mathrm{A}$. Asthma exacerbations during the first trimester of pregnancy and the risk of congenital malformations among asthmatic women. J Allergy Clin Immunol 2008;121:1379-84, 84.

16 Eltonsy S, Forget A, Blais L. Beta(2) -agonists use during pregnancy and the risk of congenital malformations. Birth Defects Res A Clin Mol Teratol 2011;91:937-47.

17 Kallen B, Rydhstroem H, Aberg A. Asthma during pregnancy-a population based study. Eur J Epidemiol 2000;16:167-71.

18 Bakhireva $\mathrm{LN}$, Jones $\mathrm{KL}$, Schatz $\mathrm{M}$, et al. Asthma medication use in pregnancy and fetal growth. J Allergy Clin Immunol 2005;116:503-9.

19 Tata LJ, Lewis SA, McKeever TM, et al. Effect of maternal asthma, exacerbations and asthma medication use on congenital malformations in offspring: a UK population-based study. Thorax 2008;63:981-7.

20 Lin S, Herdt-Losavio M, Gensburg L, et al. Maternal asthma, asthma medication use, and the risk of congenital heart defects. Birth Defects Res A Clin Mol Teratol 2009;85:161-8.

21 Perlow JH, Montgomery D, Morgan MA, et al. Severity of asthma and perinatal outcome. Am J Obstet Gynecol 1992;167(4 Pt 1):963-7.

22 Namazy JA, Murphy VE, Powell H, et al. Effects of asthma severity, exacerbations and oral corticosteroids on perinatal outcomes. Eur Respir J 2013;41:1082-90.

23 Murphy V, Wang G, Namazy J, et al. The risk of congenital malformations, perinatal mortality and neonatal hospitalisation among pregnant women with asthma: a systematic review and meta-analysis. BJOG 2013;120:812-22.

24 Maternal asthma and the risk of major congenital malformations: is drug insurance status an effect modifier? J Popul Ther Clin Pharmacol 2014;21:e308-37; 
September 17, 2014 Supplement. 4th RQRM Annual Meeting 'New Challenges in Drug Research', Orford, Québec, Canada. Abstract \#9. http://www.jptcp.com/ pubmed. php? articleld $=475$.

25 Vilain A, Otis S, Forget A, et al. Agreement between administrative databases and medical charts for pregnancy-related variables among asthmatic women. Pharmacoepidemiol Drug Saf 2008;17:345-53.

26 Gershon AS, Wang C, Guan J, et al. Identifying patients with physician-diagnosed asthma in health administrative databases. Can Respir J 2009;16:183-8.

27 Heinonen OP, Slone D, Shapiro S. Birth defects and drugs in pregnancy. Littleton: Publishing Sciences Group, Inc., 1977.

28 Blais L, Berard A, Kettani FZ, et al. Validity of congenital malformation diagnostic codes recorded in Quebec's administrative databases. Pharmacoepidemiol Drug Saf 2013;22:881-9.
29 Zeger SL, Liang KY, Albert PS. Models for Longitudinal data—a generalized estimating equation approach. Biometrics 1988;44:1049-60.

30 Greenland S. Modeling and variable selection in epidemiologic analysis. Am J Pub Hlth 1989;79:340-9.

31 Murphy VE, Clifton VL, Gibson PG. Asthma exacerbations during pregnancy: incidence and association with adverse pregnancy outcomes. Thorax 2006;61:169-76.

32 Webster WS, Abela D. The effect of hypoxia in development. Birth Defects Res C Embryo Today 2007;81:215-28.

33 Rabe KF, Atienza T, Magyar P, et al. Effect of budesonide in combination with formoterol for reliever therapy in asthma exacerbations: a randomised controlled, double-blind study. Lancet 2006;368:744-53.

34 Suissa $S$, Ernst $P$, Kezouh A. Regular use of inhaled corticosteroids and the long term prevention of hospitalisation for asthma. Thorax 2002;57:880-4. 